\title{
Raman Depolarization Ratios in RNA and DNA are Sensitive for Sugar-Base Coupling
}

\author{
P. A. TERPSTRA, C. OTTO, * G. M. J. SEGERS-NOLTEN, I. S. KANGER, and J. GREVE
}

University of Twente, Department of Applied Physics, P.O. Box 217, 7500 AE Enschede, The Netherlands

\begin{abstract}
SYNOPSIS
Polarized and depolarized Raman spectra are obtained for a number of synthetic polynucleotides containing adenine, uracil, and thymine bases. The depolarization ratios are determined by two methods: (1) by dividing the $\perp$-spectrum by the $\|$-spectrum and (2) after curve fitting. Overlapping bands, isotope splitting, reorientational broadening, and noncoincidence splitting affect the magnitude of the depolarization ratios over the bandwidth. For both Lorentz and Gauss curves these influences are simulated. A comparison of the Raman spectra of RNA and DNA molecules shows that the depolarization ratios for a number of similar base vibrations are different. The vibrational modes and the depolarization ratios of sugar vibrations are most sensitive to the structure of the polynucleotide. Base vibrations that have their potential energy distributed over base and sugar atoms also seem to be more sensitive to the structure. For instance the adenine vibrations at $1332 \mathrm{~cm}^{-1}$ and $1344 \mathrm{~cm}^{-1}$ in poly (dA), poly (dA) p poly (dT) and poly (dA$\mathrm{dT}) \cdot$ poly $(\mathrm{dA}-\mathrm{dT})$ have different depolarization ratios of, respectively, 0.36 and 0.28 . This supports a previous assignment of the $1332 \mathrm{~cm}^{-1}$ band to a different sugar pucker $\left(\mathrm{O}_{4}\right.$,-endo) than the $\mathrm{C}_{2^{\prime}}$-endo corresponding with the $1344 \mathrm{~cm}^{-1}$ vibration. Assuming equal Raman scattering coefficients for this vibration, irrespective of the sugar pucker gives rise to the following ratio of the $\mathrm{O}_{4^{\prime}}$-endo/ $\mathrm{C}_{2^{\prime}}$-endo in poly $(\mathrm{dA})$ of 0.41 , in poly $(\mathrm{dA}) \cdot \operatorname{poly}(\mathrm{dT})$ of 0.37 , and in poly $(\mathrm{dA}-\mathrm{dT}) \cdot \operatorname{poly}(\mathrm{dA}-\mathrm{dT})$ of 0.41 . (C) $1995 \mathrm{John}$ Wiley \& Sons, Inc.
\end{abstract}

\section{INTRODUCTION}

The measurement of depolarization ratio $\rho$ is useful in the assignment of vibrational bands in Raman spectra to particular motions of the nuclei. In the Raman spectra of polynucleotides most of the intense vibrations are polarized. As a consequence these vibrations have the same local symmetry as the molecular group in which they originate. Thus the ring vibrations occur in the planes of the bases and the phosphate groups involve symmetric stretching of PO bonds. ${ }^{1}$ Lord and Thomas ${ }^{2}$ reported $\rho$ of a number of intense bands in spectra of RNA. This analysis enabled the assignment of bands to particular molecular groups constituting the polynucleotides. It was reported ${ }^{3}$ that the $\rho$ of

* To whom correspondence should be addressed.

Biospectroscopy, Vol. 1, 255-263 (1995)

(C) 1995 John Wiley \& Sons, Inc.

CCC 1075-4261/95/040255-09 the uracil vibration at $1230 \mathrm{~cm}^{-1}$ was twice as large in the mononucleotide as in the single-stranded polynucleotide. No further sensitivity of the $\rho$ was reported comparing mononucleotides and singlestranded polyribonucleotides.

Measurements on polynucleotides oriented both in a hydrodynamic flow ${ }^{4}$ and in single crystals ${ }^{5}$ showed that the off-diagonal elements of the various Raman tensors are much smaller than the diagonal elements. For the strong base vibrations, the $\alpha_{x x}$ and $\alpha_{y y}$ were found to be 10 times larger than the $\alpha_{z z}$, where $z$ is the direction of the helix axis. The dependence on the scattering geometry was large for the base vibrations and small for the sugar groups, suggesting that the latter were not well oriented. The observed $\rho$ were used to identify different vibrational modes.

Recently the $\rho$ of Raman bands in the spectra of mononucleotides, dissolved in protonated and deuterated water, were reported. The relative magni- 
tude of the components of the Raman scattering tensor can be estimated, using a reasonable, but simplified, tensorial representation. ${ }^{6}$ For the base vibrations it was assumed that $\alpha_{z z}$ is perpendicular to the base plane. From this the relative magnitude for $\alpha_{x x}$ and $\alpha_{y y}$ could be estimated for some vibrations. Benevides et al. ${ }^{7}$ expanded this study to a double-helical guanine and cytosine containing Z-DNA and obtained relations for the magnitudes of the Raman tensor components, which helped the assignment of some sugar modes. The relative orientation of various residues in a sugar-phosphatebase conformer could be proposed on the basis of the measurement of the orientation of the Raman tensors.

The $\rho$ reported in the literature for Raman bands of (poly) nucleotides has been obtained by dividing peak amplitudes. However, overlapping bands, isotope splitting, reorientational broadening and noncoincidence splitting affect the magnitude of the $\rho$. In this article the spectral dependence of the $\rho$ is compared with the $\rho$ obtained after curve fitting both the $\perp$ - and $\|$-Raman spectrum. The results obtained by the latter method are more accurate and are used to show that the $\rho$ of the base vibrations that have their potential energy distributed over the sugar group change with changes in the secondary structure.

\section{MATERIALS AND METHODS}

From Sigma we purchased the mononucleotides rAMP (Lot no. 90H7215), dAMP (lot no. 66C-7040) and rUMP (lot no. 48F05481). All polynucleotides were purchased from Pharmacia (poly[rA], lot no. AA4110P01; poly [dA], lot no. 3017836011; poly [rU], lot no. AB4440P08; poly [rA] ·poly [rU], lot no. QH82452101; poly [dA] poly [dT], lot no. 2107860011; and poly [dA-dT] - poly [dA-dT], lot no. AE7870108). The nucleotides were dissolved in an aqueous buffer solution containing $93 \mathrm{mM} \mathrm{NaCl}, 10 \mathrm{mM} \mathrm{Na} \mathrm{NPO}_{4}$, $7 \mathrm{mM}$ NaCacodylate, and the $\mathrm{pH}$ of the buffer was 7.2. The concentrations were $78 \mathrm{mM}$ for $\mathrm{rAMP}$ and rUMP, and $20 \mathrm{mM}$ for dAMP. The concentrations for the single-stranded polynucleotides were $78 \mathrm{mM}$ bases for poly (rA) and poly (rU) and $14 \mathrm{mM}$ bases for poly (dA). The double-stranded polynucleotides were dissolved in the concentrations of $14 \mathrm{mM}$ base pairs for poly ( $\mathrm{rA}) \cdot$ poly $(\mathrm{rU})$ and $10 \mathrm{mM}$ base pairs for poly $(d A-d T) \cdot p o l y(d A-d T)$ and poly $(d A)$ $\cdot \operatorname{poly}(\mathrm{dT})$.

The Raman spectrometer consisted of an $\mathrm{Ar}^{+}$ ion laser (Spectra-Physics 2025-03), a double monochromator (Jobin-Yvon HG2S), and a Hamamatsu R943-02 photomultiplier. A 90 scattering geometry was used. The scattered light passes through an analyzer (Spindler \& Hoyer, 10K) with a suppression ratio of $10^{-4}$. The accuracy of the measurement of the $\rho$ is improved by quasisimultaneously measuring the $\|$ - (vertically polarized excitation and vertically polarized scattered light) and $\perp$ - (horizontally polarized excitation and vertically polarized scattered light) spectra. At each frequency, the vertical component of the signal was recorded with and without a $\lambda / 2$ plate in the excitation path; hence corresponding spectral points in the $\|$ - and $\perp$-spectra are obtained at exactly the same monochromator position. The $\rho$ of the 667 $\mathrm{cm}^{-1}$ band in chloroform was determined to be 0.011 . This is smaller than the value of 0.014 previously reported, ${ }^{8}$ indicating that the polarization purity both in excitation and emission is of good quality.

The slitwidths of the monochromator were set to $400 \mu \mathrm{m}$. The resolution of the setup was determined from the response to the $\mathrm{Ar}^{+}$-ion laser emission at $514.5 \mathrm{~nm}$ and the $\mathrm{He}-\mathrm{Ne}$ laser emission at $632.8 \mathrm{~nm}$. The setup response was well described, with a Gaussian convolution with a bandwidth of 3 $\mathrm{cm}^{-1}$. All the spectra were deconvoluted with the setup response.

The transmission and detection sensitivity of the setup was characterized with the emission of a tungsten lamp. The deviation of the response from the emission curve of the black-body radiator was used for intensity calibration. The excitation wavelength used was $514.5 \mathrm{~nm}$.

The spectra for rAMP, rUMP, poly (rA), and poly ( $r U)$ were recorded with 15 seconds per spectral point. The spectra of the double-stranded nucleotides were recorded with 55 seconds per spectral point.

The amplitude depolarization ratios are calculated as the quotient of the $\perp$ - and \|-spectra, both corrected for buffer and residual background contributions. The error in this amplitude $\rho$ is dependent on the resultant Poisson noise in the spectra. This error has been calculated for two adenine vibrations in poly ( $\mathrm{dA})$ : the almost completely polarized band at $727 \mathrm{~cm}^{-1}$ and the partially depolarized band at $1344 \mathrm{~cm}^{-1}$. The $727 \mathrm{~cm}^{-1}$ adenine vibration is a polarized band in a spectral region with a large background from the buffer. This gives rise to a maximal error. The measurement error in amplitude $\rho$ has been calculated to be 5\%. For a depolarized band ( $\rho=0.28)$, such as the $1344 \mathrm{~cm}^{-1}$ band in adenine, the error is calculated as $1 \%$. 


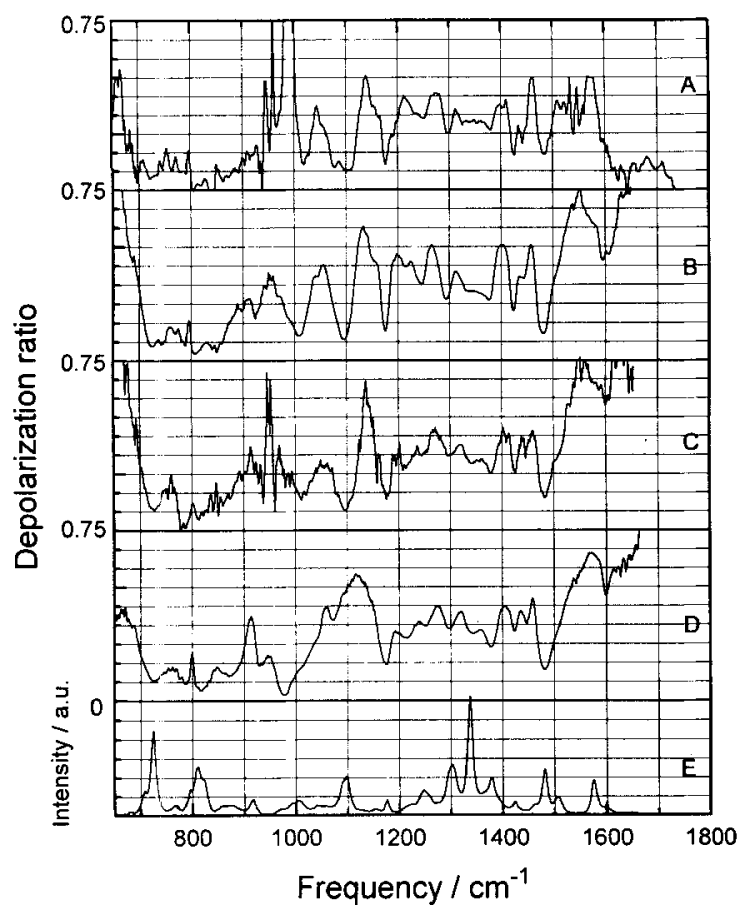

Figure 1. Amplitude depolarization ratios for adenine containing ribonucleotides: (A) poly (rA).poly (rU); (B) poly (rA ); (C) poly (rA), $75^{\circ} \mathrm{C}$; (D) rAMP; and (E) $\|$-spectrum of poly (rA).

The RAMPAC program was used to perform the data analysis and the curve fitting. This program is described by de Mul and Greve. ${ }^{9}$ The measured spectra were corrected for buffer contributions. Spectra of double-stranded structures were also corrected for a small residual background on the low-wavenumber side.

\section{RESULTS}

The spectral dependence of the $\rho$ is obtained by dividing the experimental $\perp$ - and $\|$-spectra and reading the $\rho$ at the frequency of the peak maximum. This will be called the amplitude $\rho$. The amplitude $\rho$ depends on the estimate for the zero-scattering level in both spectra. In Figures 1 and 2 the data are shown for ribonucleotides containing only adenine and uracil. Figure 3 shows the data for deoxyribonucleotides containing only adenine and thymine. Our amplitude $\rho$ are on the average 0.03 larger than the values of Ueda, ${ }^{6}$ which is possibly due to differences in this estimate of the background.

From the data of Figures 1 to 3, a quick overview of the changes can be obtained. Changes in the $\rho$ occur in the frequency region between $800 \mathrm{~cm}^{-1}$

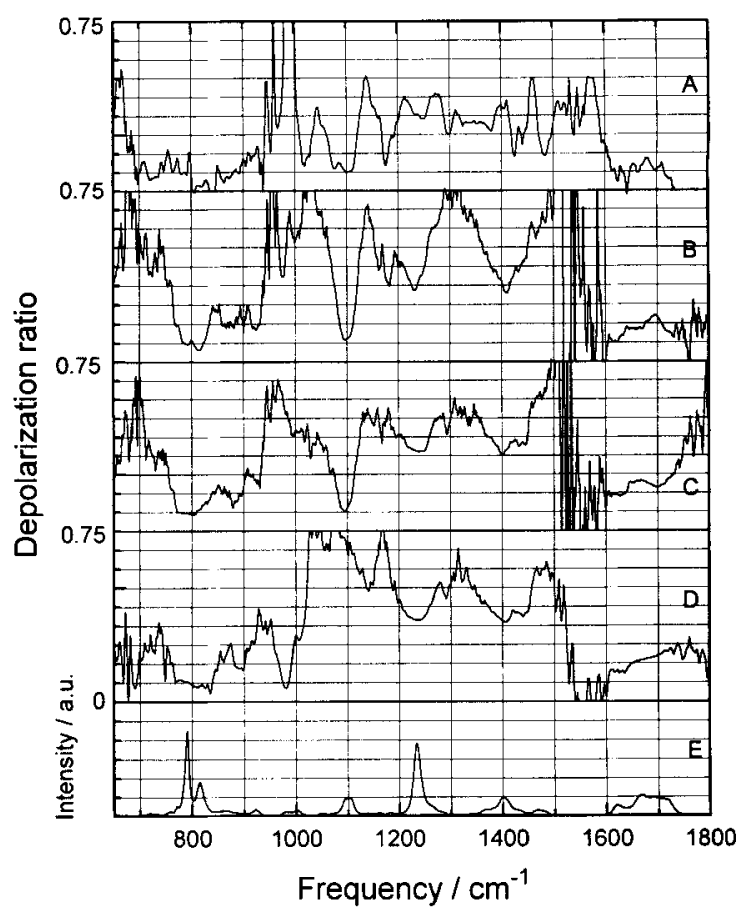

Figure 2. Amplitude depolarization ratios for uracilcontaining ribonucleotides. (A) poly $(\mathrm{rA}) \cdot \operatorname{poly}(\mathrm{rU})$; (B) poly (rU); (C) poly (rU), $45^{\circ} \mathrm{C}$; (D) rUMP; and (E) $\|$-spectrum of poly $(\mathrm{rU})$.

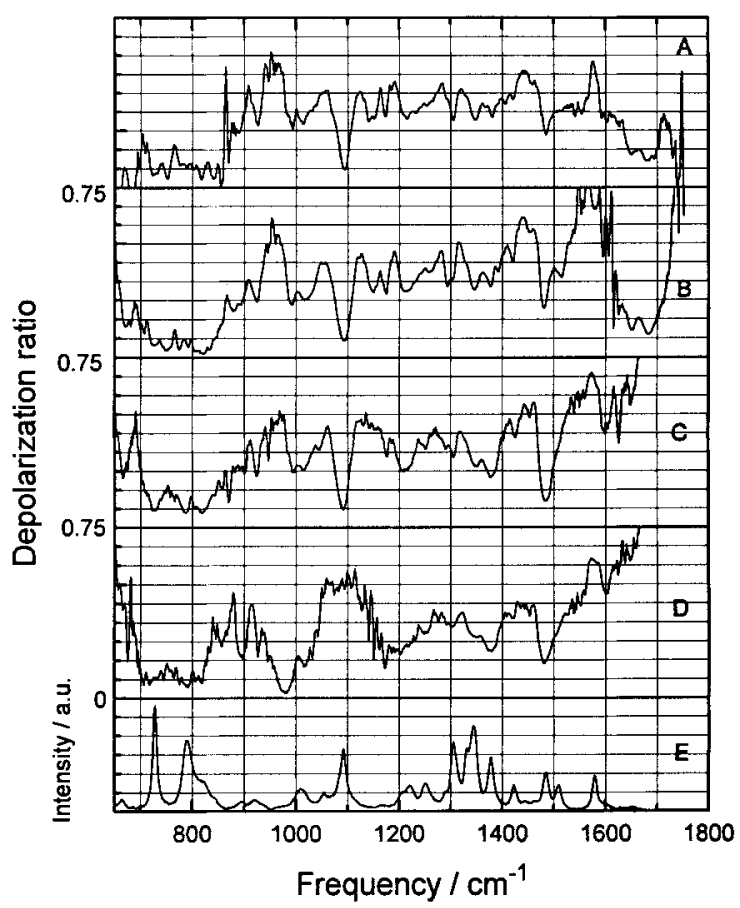

Figure 3. Amplitude depolarization ratios for adenine-containing deoxyribonucleotides: (A) poly(dA)-poly (dT); (B) poly (dA-dT) - poly (dA-dT); (C) poly(dA); (D) dAMP; and (E) \|-spectrum of poly (dA). 
A

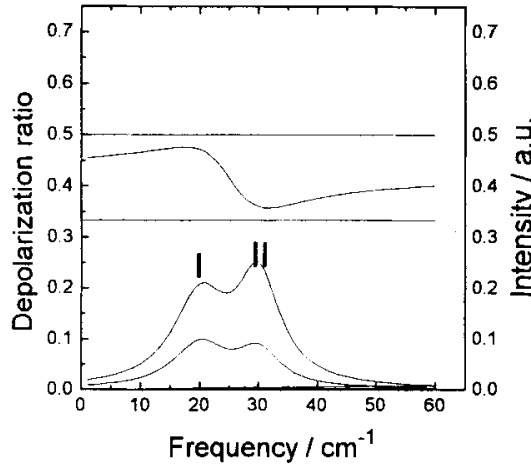

B

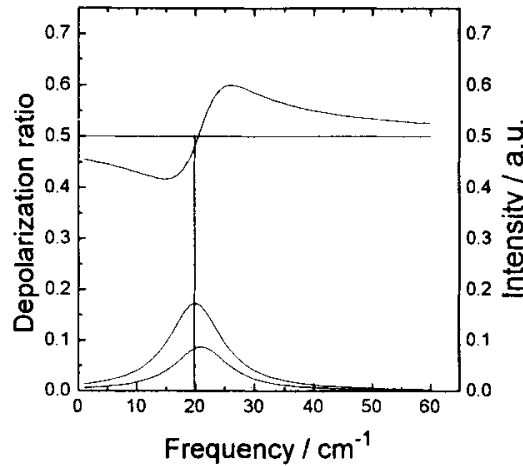

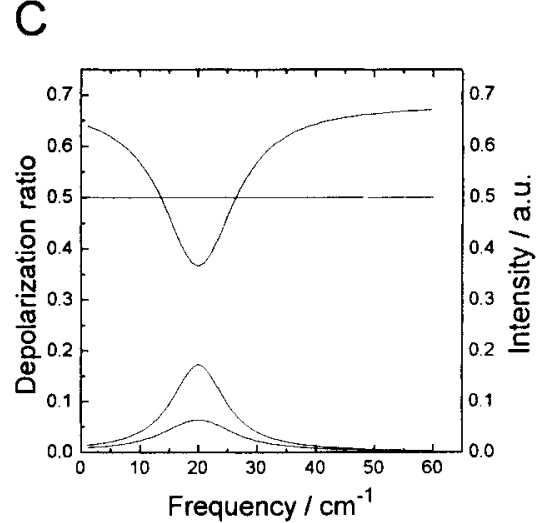

Figure 4. The effects of (A) overlapping bands, (B) noncoincidence splitting, and (C) reorientational broadening on the amplitude depolarization ratio for Lorentz bands. (A) Band I: $\rho=0.5, \omega_{\max }=20 \mathrm{~cm}^{-1}, \Delta \omega=11 \mathrm{~cm}^{-1}$; band II: $\rho=0.333, \omega_{\max }=30 \mathrm{~cm}^{-1}, \Delta \omega=9$ $\mathrm{cm}^{-1}$. (B) $\rho=0.5, \omega_{\max }^{\|}=20 \mathrm{~cm}^{-1}, \omega_{\max }^{\perp}=21 \mathrm{~cm}^{-1}, \Delta \omega=11 \mathrm{~cm}^{-1}$, (C) $\rho=0.5, \omega_{\max }=20$ $\mathrm{cm}^{-1}, \Delta \omega^{\perp}=11 \mathrm{~cm}^{-1}, \Delta_{\omega}^{\perp}=15 \mathrm{~cm}^{-1}$.

and $1200 \mathrm{~cm}^{-1}$. Mainly the sugar and phosphate groups contribute here. The spectra of the mononucleotides show the $\mathrm{PO}_{3}^{2-}$ vibration at $980 \mathrm{~cm}^{-1}$, whereas the spectra of the polynucleotides have the $\mathrm{PO}_{2}^{-}$vibration at $1100 \mathrm{~cm}^{-1}$.

Changes in $\rho$ can quickly be obtained from these figures; however the spectral dependence of the $\rho$ is influenced by the choice of the zero-scattering level, overlapping bands with different $\rho$, reorientational broadening of the $\perp$-spectrum, and noncoincidence splitting. ${ }^{10,11}$ The accuracy with which $\rho$ can be obtained depends on the intensity in both the $\perp$ - and the $\|$-spectrum. Most important is the appropriate choice of the background in the $\perp$ spectrum, being the weaker of the two. In a curve fit the background is a result of the overlap of the wings of the Lorentz curves. The physical level of zero scattering is not on the level of the minima in the spectrum, but lies below this level, thereby increasing the intensity in the spectra.

Apart from the estimate of the background all mentioned effects depend on the bandshape. In Figure $4 \mathrm{~A}$ we show the effect of overlapping Lorentz bands with different $\rho$. Isotope splitting is considered as a case of overlapping bands. The resulting amplitude $\rho$ are a mix of the physical values and the physical $\rho$ are not obtained at any frequency position. The resulting error depends predominantly on the band separation relative to the bandwidths. Figure 4B illustrates the effect of noncoincidence splitting. A realistic value of $1 \mathrm{~cm}^{-1}$ is chosen ${ }^{10,11}$ and the vibrational bandwidth is 11 $\mathrm{cm}^{-1}$. In Figure $4 \mathrm{C}$ the effect of a reorientational broadening of $4 \mathrm{~cm}^{-1}$ on a bandwidth of $11 \mathrm{~cm}^{-1}$ is shown for a Lorentz bandshape. The actual $\rho$ is 0.5 , but it is observed that the $\rho$ varies appreciably over the bandwidth. In Figure 5A, 5B, and 5C the same cases are shown for Gauss bandshapes, again showing a complex frequency dependence of the $\rho$. One observes that the $\rho$ has significant dispersion over the bandwidth and, hence, that the value of the depolarization ratio cannot simply be obtained from the spectral dependence. In order to illustrate the effect in an experimental case, the $\perp / \|$-spectrum of $\mathrm{CHCl}_{3}$ (Figure 6) was simulated. Introducing the effects of reorientational broadening and neighbouring bands gives a proper reconstruction of the spectrum of the $\rho$. The increase at lower frequencies and the decrease at the higher frequencies are caused by overlap of the two vibrational bands. The $\rho$ of 0.008 at the peak maximum at $667 \mathrm{~cm}^{-1}$ is the result of reorientational broadening. The real value is 0.011 . The decrease by reorientational broadening is partly compensated by an increase due to the overlap with the anisotropic band at 760 $\mathrm{cm}^{-1}$. The experimental amplitude $\rho$ shows a dip at $650 \mathrm{~cm}^{-1}$, which is caused by a weak, polarized band at this frequency. It is also concluded from this experimental result that the actual $\rho$ cannot simply be obtained from the spectral dependence of the $\rho$; a curve-fit analysis is necessary to obtain physical $\rho$ values.

The $\rho$ based on the intensities from the curve fit has been obtained for the nucleotide vibrations. The $785-\mathrm{cm}^{-1}$ uracil vibration has a $\rho$ of 0.07 , and no changes could be observed as a function of the secondary structure. The previously reported ${ }^{3}$ difference in $\rho$ for the $1230 \mathrm{~cm}^{-1}$ between $\mathrm{rUMP}$ 
A

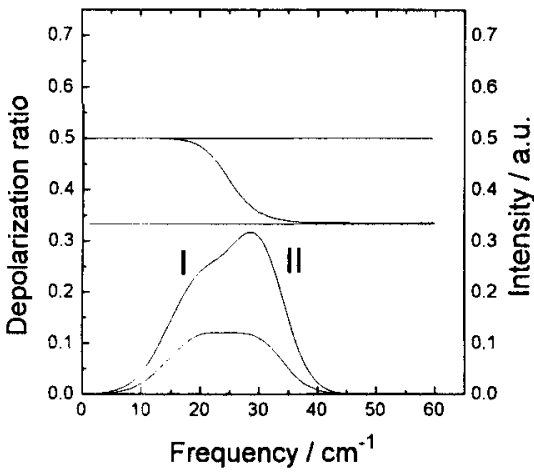

B

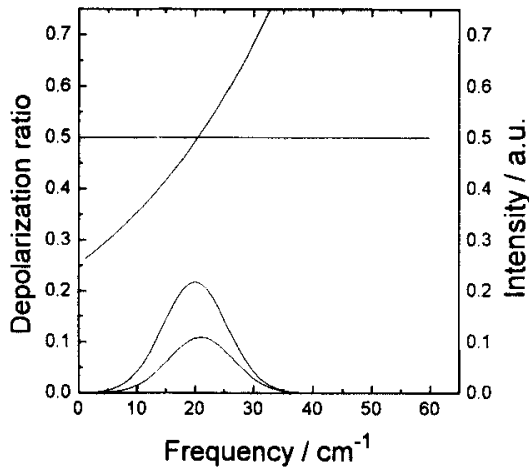

C

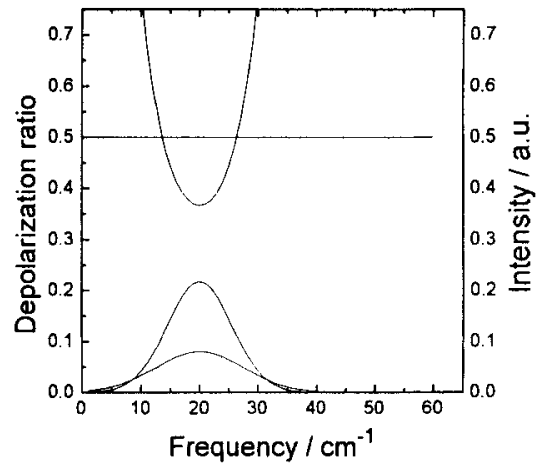

Figure 5. The effects of (A) overlapping bands, (B) noncoincidence splitting, and (C) reorientational broadening on the amplitude depolarization ratio for Gauss bands. (A) Band I: $\rho=0.5, \omega_{\max }=20 \mathrm{~cm}^{-1}, \Delta \omega=11 \mathrm{~cm}^{-1}$; band II: $\rho=0.333, \omega_{\max }=30 \mathrm{~cm}^{-1}, \Delta \omega=9$ $\mathrm{cm}^{-1}$. (B) $\rho=0.5, \omega_{\max }^{\prime \prime}=20 \mathrm{~cm}^{-1}, \omega_{\max }^{\perp}=21 \mathrm{~cm}^{-1}, \Delta \omega=11 \mathrm{~cm}^{-1}$. (C) $\rho=0.5, \omega_{\max }=20$ $\mathrm{cm}^{-1}, \Delta \omega^{\|}=11 \mathrm{~cm}^{-1}, \Delta \omega^{\perp}=15 \mathrm{~cm}^{-1}$.

and poly $(\mathrm{rU})$ is not in agreement with our data. The $\rho$ is 0.32 in both cases. In Figure $7 \mathrm{~A}-\mathrm{F}$ the $\rho$ of the strong adenine base vibrations has been compared for different secondary structures. The $\rho$ of the $727 \mathrm{~cm}^{-1}$ is small and no significant changes

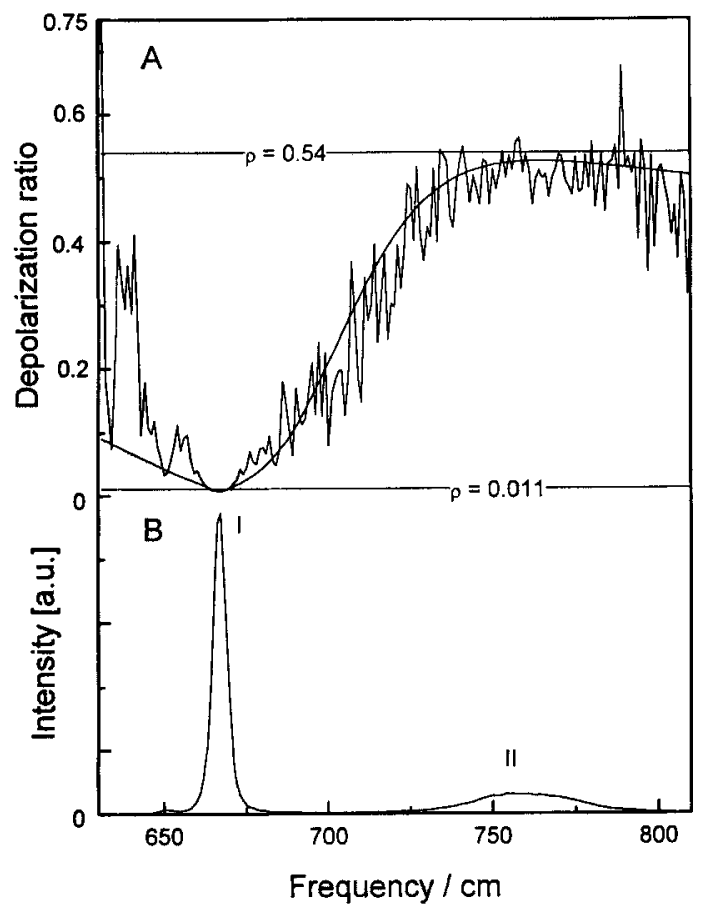

Figure 6. (A) Amplitude depolarization ratio for chloroform $(\perp / \|)$. Band I: $\rho=0.011, \omega_{\max }=667 \mathrm{~cm}^{-1}$, $\Delta \omega^{\|}=5 \mathrm{~cm}^{-1}, \Delta \omega^{\perp}=8.5 \mathrm{~cm}^{-1}$; band II: $\rho=0.54, \omega_{\max }$ $=760 \mathrm{~cm}^{-1}, \Delta \omega=38 \mathrm{~cm}^{-1}$. (B) Total Raman spectrum of choroform $(\|+1)$. occur that are larger than the error in $\rho$. The $\rho$ of the band of adenine at $1378 \mathrm{~cm}^{-1}$ does not depend on the type of sugar in the nucleotide or on the conformation of the sugar group in the polynucleotide. The adenine band at $1305 \mathrm{~cm}^{-1}$ can be compared between rAMP, unstacked poly (rA), dAMP, and poly (dA). No differences are observed. In stacked poly ( $\mathrm{rA}$ ) this band is split in two components and in double-stranded structures overlap occurs with uracil or thymine modes.

On the other hand, some adenine base vibrations show different $\rho$ in different structures. The $1480 \mathrm{~cm}^{-1}$ adenine vibration has a $\rho$ of 0.18 in double-stranded structures and 0.09 in single-stranded structures and mononucleotides. The $1575 \mathrm{~cm}^{-1}$ has a $\rho$ that is 0.1 lower in double-stranded RNA than in single-stranded RNA and RNA mononucleotides. This difference could not be observed in DNA. The $\rho$ of the $1506-\mathrm{cm}^{-1}$ adenine vibration changes with a change in order of the helical structure. In mononucleotides and unstacked poly ( $\mathrm{rA}$ ), the $\rho$ is $0.1-0.13$ lower than in stacked structures. The vibration at $1423 \mathrm{~cm}^{-1}$ is more intense in deoxyribonucleotides than in ribonucleotides. The depolarization ratio of this band is 0.5 in doublestranded and single-stranded polydeoxynucleotides and also in dAMP. In polyribonucleotides the depolarization ratio is approximately 0.2 and approximately 0.3 for rAMP, as can be observed from the amplitude data (Figures 1 and 3). The 1220$\mathrm{cm}^{-1}$ vibration changes in $\rho$ upon melting of singlestranded poly (rA). In stacked poly (rA) the $\rho$ is 0.45 , whereas in rAMP and unstacked poly $(\mathrm{rA})$ it is 0.25 . In poly ( $\mathrm{dA}$ ) the $\rho$ is 0.24 , and in dAMP it is 

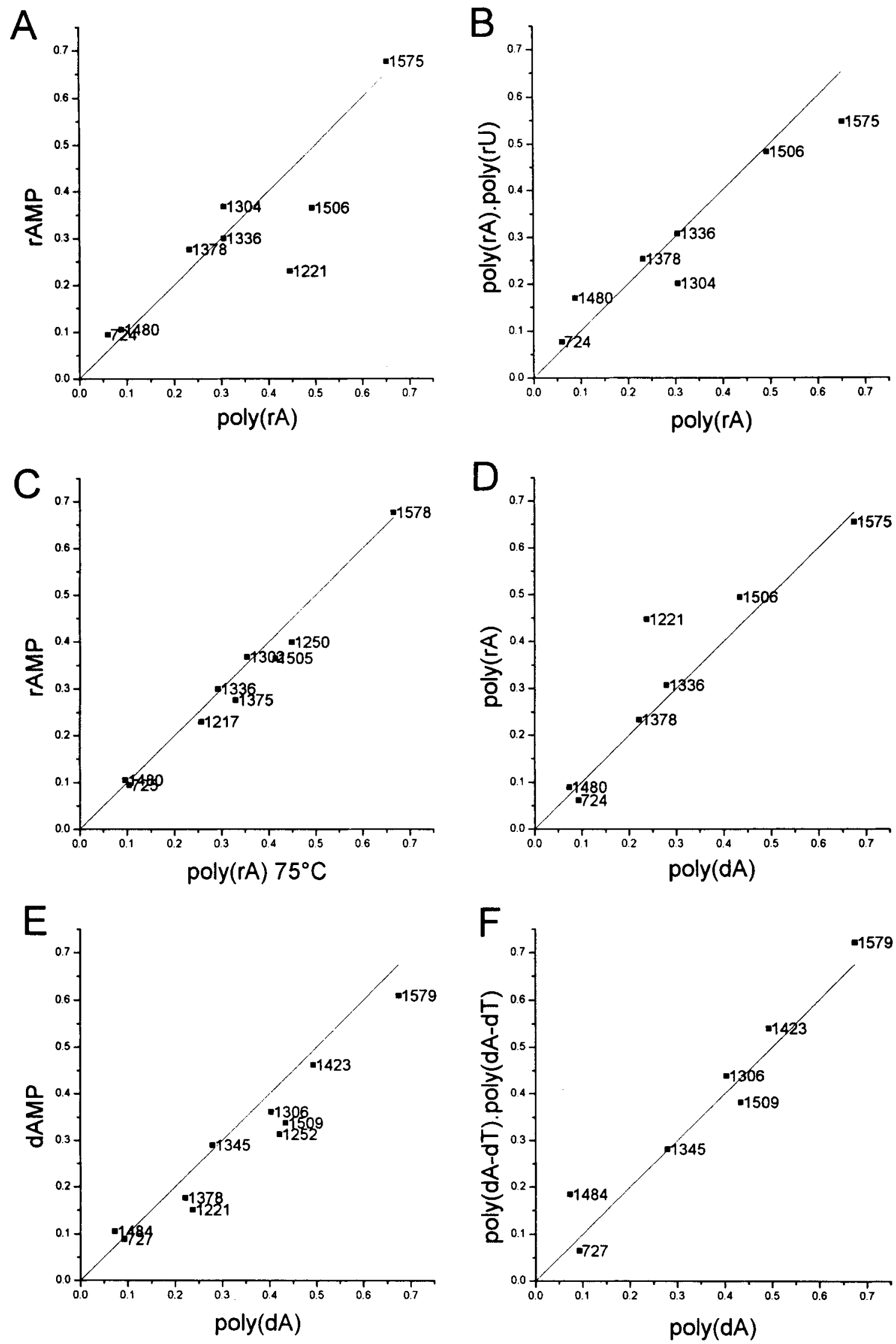

Figure 7. Comparison of the intensity depolarization ratios of adenine vibrations for different nucleotides. 


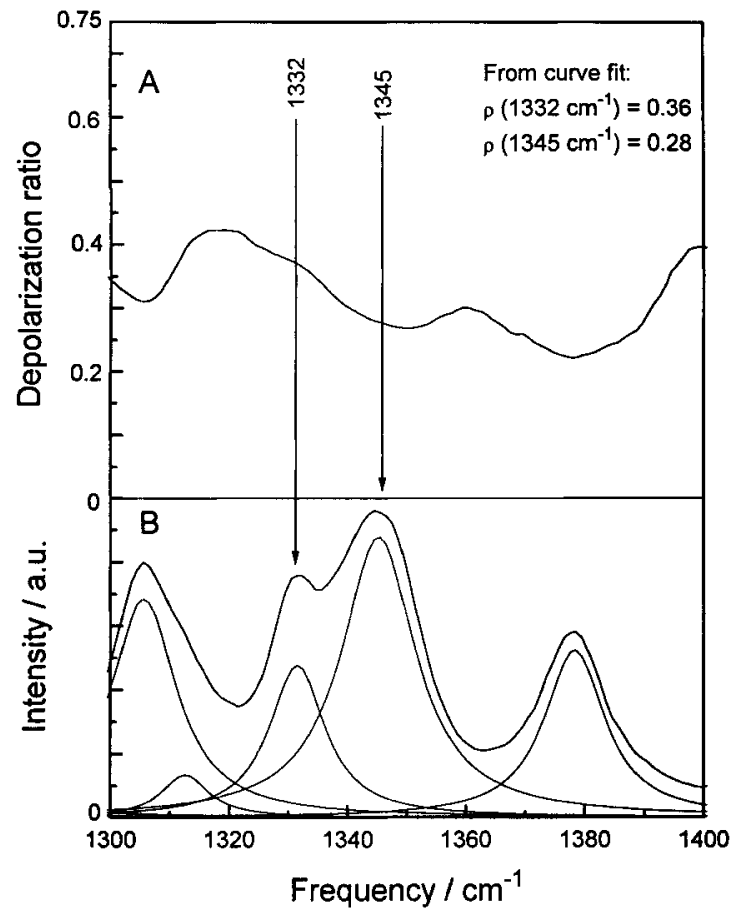

Figure 8. (A) Dependence of the depolarization ratio of the adenine vibration around $1336 \mathrm{~cm}^{-1}$ on the sugar pucker in poly (dA). Different sugar pucker shift the adenine vibration. An $\mathrm{O}_{4}$-endo sugar pucker is characterized by an adenine vibration at $1332 \mathrm{~cm}^{-1}$ with a $\rho$ of 0.36 and a $\mathrm{C}_{2^{\prime}}$-endo sugar pucker by an adenine vibration at $1345 \mathrm{~cm}^{-1}$ with a $\rho$ of 0.28 . (B) Total Raman spectrum of poly $(\mathrm{dA})(\|+\perp)$.

0.15 . This band is however overlapped by another vibration at $1204 \mathrm{~cm}^{-1}$, which makes the fit error as large as 0.05 . The difference between stacked poly (rA) and the other structures, however, is larger than this error and this difference is observable also from the amplitude $\rho$ in Figures 1 and 3 . In double-stranded structures, this vibration is obscured due to strong uracil and thymine vibrations. In Figure 8, the vibrational bands around 1336 $\mathrm{cm}^{-1}$ of poly (dA) are shown with the amplitude $\rho$ spectrum. In DNA the $1336-\mathrm{cm}^{-1}$ adenine vibration of RNA splits in two components, with frequencies of $1332 \mathrm{~cm}^{-1}$ and $1344 \mathrm{~cm}^{-1}$. It is observed from both the amplitude data and the curve-fit results that the $\rho$ of these two vibrations are different.

\section{DISCUSSION}

In Raman spectra of polynucleotides many overlapping bands occur. Curve fitting these spectra will give rise to accurate band parameters if the bands are separated in the order of their bandwidths. The error in the $\rho$, which is defined as the quotient of two intensities measurements, will be larger if the $\rho$ is close to zero, due to the uncertainty in the intensity of the $\perp$-spectrum. The error is smaller than $25 \%$ for the bands with a $\rho$ smaller than 0.1 , for instance for the bands at $724 \mathrm{~cm}^{-1}$ and $1480 \mathrm{~cm}^{-1}$. Most of the bands in the Raman spectrum have a $\rho$ much larger than 0.1. For these bands the error in the $\rho$ is smaller than $10 \%$, becoming 5\% for intense depolarized bands. The depolarization ratio of the base vibrations is shown in Figure 7.

The sugar-phosphate vibrations have a low intensity and a large bandwidth. The observed differences in $\rho$ are large enough to be observed with amplitude data. In Figure 9, the sugar-phosphate vibrations are compared for poly $(\mathrm{rA})$ and poly ( $\mathrm{dA}$ ). The $1100 \mathrm{~cm}^{-1} \mathrm{PO}_{2}^{-}$vibration does not change in $\rho$ with changes in the secondary structure. However, large differences (up to $100 \%$ ) can be observed for the vibrations of the sugar-phosphate bonds. A mechanical coupling of the atomic motions in the base and the sugar has been shown to exist, ${ }^{12}$ leading to a distribution of the potential

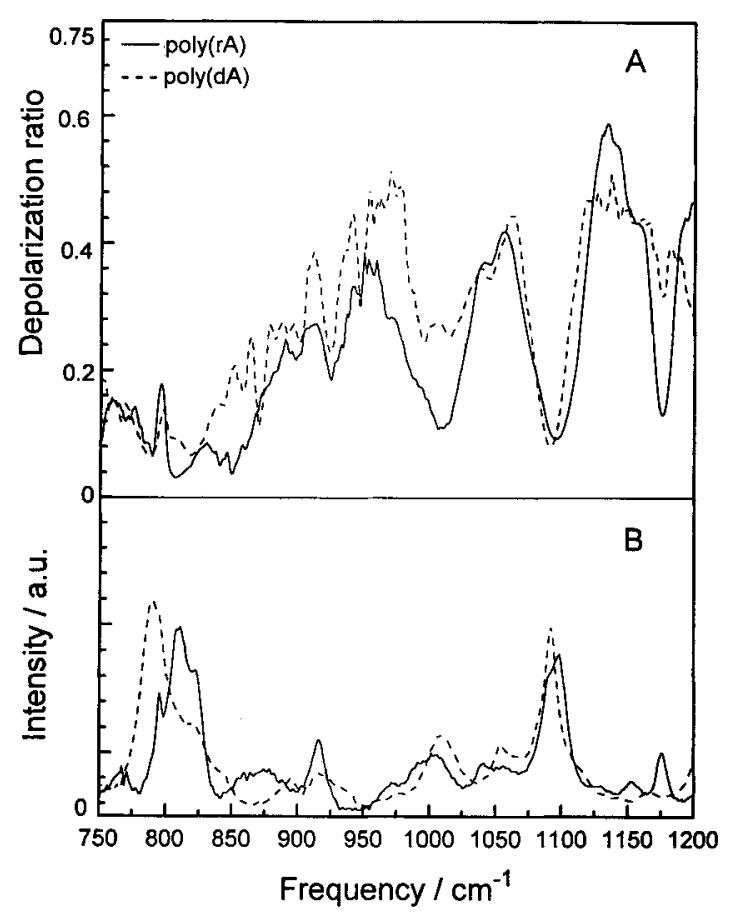

Figure 9. Sugar phosphate vibrations have a different depolarization ratio and frequency in poly (rA) and poly (dA). (A) Amplitude depolarization ratios $(\perp / \|)$. (B) Total Raman spectra of poly (rA) and poly(dA) $(\|+\perp)$. 
energy of the vibrational mode. This may explain the observed changes in $\rho$ for some base vibrations. Letellier et al. ${ }^{12}$ showed, with normal mode calculations, that the oscillator frequencies of a number of base vibrations are sensitive to the glycosidic bond angle. It was calculated that in the anticonformation the frequencies of the $1423 \mathrm{~cm}^{-1}$ and $1480 \mathrm{~cm}^{-1}$ vibrations were most sensitive. The depolarization ratio of the band at $1480 \mathrm{~cm}^{-1}$ is sensitive to conformational changes (Figure 7 ), and in particular to double-stranded formation. The band at $1423 \mathrm{~cm}^{-1}$ in deoxyribonucleotides has been assigned as partially due to the $2^{\prime} \mathrm{CH}_{2}$ scissoring mode of the deoxyribosyl group. ${ }^{13}$ Also in ribonucleotides a band at this position is present with comparable bandwidth, however, with a reduced intensity. As the $2^{\prime} \mathrm{CH}_{2}$ group has been replaced by the $2^{\prime} \mathrm{CHOH}$, it is assumed that this band originates in the base residue. We find, experimentally, that the depolarization ratio of this base vibration depends on the type of sugar molecule. We therefore propose that the depolarization ratios of some base vibrations are connected to changes in the structure of the sugar-base conformer.

The Raman spectra of poly (dA), poly (dA)- poly $(\mathrm{dT})$, and poly $(\mathrm{dA}-\mathrm{dT}) \cdot \operatorname{poly}(\mathrm{dA}-\mathrm{dT})$ show a number of indications for structural inhomogeneity. ${ }^{14-16}$ In the spectra of all these molecules a double-band structure appears with the positions at $1332 \mathrm{~cm}^{-1}$ and $1344 \mathrm{~cm}^{-1}$. Comparison of the spectra of poly (rA) and poly ( $\mathrm{dA})$ shows that the sum of the total intensities $\left(I_{\|}+I_{\perp}\right.$ ) of the two components in poly $(\mathrm{dA})$ and the total intensity of the band at $1336 \mathrm{~cm}^{-1}$ in poly (rA) are comparable within $5 \%$. It is reasonable to assume that in poly (dA) a single band has split in two bands as a result of the coupling of the adenine base vibrations to more than one sugar pucker, rather than the appearance of a totally new vibration. With molecular dynamics simulations, Brahms et al. ${ }^{15}$ proposed a dynamic sugar-pucker structure, in which the sugar pucker of the adenine base is $71 \%$ of the time $\mathrm{C}_{2^{\prime}}$-endo and $29 \%$ of the time $\mathrm{O}_{4^{\prime}}$-endo for poly $(d A)$. poly $(d T)$. The results for poly (dA$\mathrm{dT}$ ) - poly (dA-dT) were similar. Brahms et al. assigned the $1344 \mathrm{~cm}^{-1}$ vibration to adenine coupled to the $\mathrm{C}_{2^{-}}$-endo sugar pucker and the $1332 \mathrm{~cm}^{-1} \mathrm{vi}-$ bration to an adenine coupled to the $\mathrm{O}_{4^{\prime}}$-endo sugar pucker. This last assignment of the $1332 \mathrm{~cm}^{-1}$ adenine vibration was made on the basis of the molecular dynamics results. In poly (rA) the $1336 \mathrm{~cm}^{-1}$ adenine vibration is coupled to a $\mathrm{C}_{3}$-endo sugar pucker and the $\rho$ is 0.30 . The much larger $\rho$ of 0.36 of the $1332 \mathrm{~cm}^{-1}$ adenine vibration in polydeoxyri- bonucleotides indicates that this vibration is not coupled to the $\mathrm{C}_{3}$-endo sugar pucker. The amplitude depolarization ratios of the bands at 1332 $\mathrm{cm}^{-1}$ and $1344 \mathrm{~cm}^{-1}$ are different by 0.09 (Fig. 8). A discussion of the error based on the noise analysis of the measured intensities is given above. The occurrence of two different sugar puckers is in agreement with the molecular dynamics results of Brahms et al. Their results, on the basis of molecular dynamics calculations, indicated the presence of an $\mathrm{O}_{4}$-endo sugar pucker together with a $\mathrm{C}_{2^{\prime}}$ endo pucker. A different conclusion was reached by Chandrasekaran et al., ${ }^{17}$ who interpreted from their $\mathrm{x}$-ray data $\mathrm{C}_{1}$-exo sugar pucker for adenine in poly $(d A)$. poly $(d T)$. The total energy of the sugar puckering as a function of the pseudorotation angle shows ${ }^{18}$ that the energy of the $\mathrm{C}_{1}$-exo pucker is intermediate between that of $\mathrm{O}_{4^{\prime}}$-endo and $\mathrm{C}_{2^{\prime}}$ endo. We surmise here that the conclusion reached by Chandrasekaran could well be in agreement with the result of Brahms et al., as the x-ray data present the time-averaged structure. We assign, following Brahms et al., the $1332 \mathrm{~cm}^{-1}$ to the $\mathrm{O}_{4^{\prime}}$ endo sugar pucker. The relative occurrence of the sugar puckers coupled to the adenine has been calculated with the relative total intensities in the $\mathrm{Ra}$ man spectra $\left(I_{\|}+I_{\perp}\right)$, assuming similar Raman cross sections. The $\mathrm{O}_{4}$,-endo $/ \mathrm{C}_{2}$,-endo intensity ratios are for poly $(\mathrm{dA})$, poly $(\mathrm{dA}) \cdot \operatorname{poly}(\mathrm{dT})$ and poly $(\mathrm{dA}-\mathrm{d} \mathrm{T})$ - poly $(\mathrm{dA}-\mathrm{d} \mathrm{T})$, respectively, 0.41, 0.37, and 0.41 . This is in agreement with residence times calculated with molecular dynamics by Brahms et al. ${ }^{15}$ for poly $(\mathrm{dA})$-poly $(\mathrm{dT})$ and poly $(\mathrm{dA}-\mathrm{dT})$ - poly (dA-dT).

\section{CONCLUSIONS}

We have shown that the amplitude $\rho$ of a typical Raman spectrum is influenced by overlapping bands, reorientational broadening, and noncoincidence splitting. The spectral dependence of the $\rho$ of $\mathrm{CHCl}_{3}$ can be fully described, taking into account the correct values for the relevant parameters. The depolarization ratios can also be determined from integrated intensities obtained by curve fitting the spectra. This method works particularly well in spectral regions where the number of bands can be determined. The curve fit results in a more accurate estimate of the "zero-scattering" level. The relative change in the magnitude of the signal is larger in the $\perp$-spectrum, which is weaker than the $\|$-spectrum. Consequently the depolarization ratios obtained from integrated intensities are larger 
than those obtained from the amplitude depolarization ratio.

It was found that the values of the $\rho$ of the adenine vibrations at $\sim 1336 \mathrm{~cm}^{-1}, 1423 \mathrm{~cm}^{-1}$, and $1480 \mathrm{~cm}^{-1}$ are sensitive to changes in the secondary structure. It is proposed that observed changes are due to coupling to the sugar-phosphate group. In adenosine ribose phosphates a $\mathrm{C}_{3}$-endo sugar pucker at $1336 \mathrm{~cm}^{-1}$ is found to be accompanied by a $\rho$ of 0.3 , while in adenosine deoxyribose a $\mathrm{C}_{2^{\prime}}$-endo sugar pucker at $1344 \mathrm{~cm}^{-1}$ and band at $1332 \mathrm{~cm}^{-1}$ give rise to $\rho$ of 0.28 and 0.36 , respectively. A previous study ${ }^{15}$ assigned the band at $1332 \mathrm{~cm}^{-1}$ to adenine residues connected to sugar residues with an $\mathrm{O}_{4}$-endo pucker. This supports the results from this study that the depolarization ratio of base vibrations may depend on the conformation of the sugar residue. A further comparison between the residence times obtained from molecular dynamics calculations ${ }^{15}$ and the Raman intensities $\left(I_{\|}+I_{\perp}\right)$ give corresponding occurrences of different sugar puckers in poly $(\mathrm{dA})$, poly $(\mathrm{dA}) \cdot \operatorname{poly}(\mathrm{dT})$, and poly $(\mathrm{dA}-\mathrm{dT}) \cdot(\mathrm{dA}-\mathrm{dT})$. The $\mathrm{O}_{4^{\prime}}$-endo $/ \mathrm{C}_{2^{\prime}}$-endo ratios are $0.41,0.37$, and 0.41 , respectively.

This work was supported by the Dutch Organisation for Scientific Research (NWO).

\section{REFERENCES}

1. K. A. Hartman, R. C. Lord, and G. J. Thomas, Jr., "Structural studies of nucleic acids and polynucleotides by infrared and Raman spectroscopy," in Physicochemical Properties of Nucleic Acids, Vol. 2, J. Duchesne (Ed.), Academic Press, London, 1973.

2. R. C. Lord and G. J. Thomas, Jr., "Raman spectral studies of nucleic acids and related molecules, I: ribonucleic acid derivatives," Spectrochim. Acta, 23A, 2551 ( 1967).

3. B. Fanconi, B. Tomlinson, L. A. Nafie, W. Small, and W. L. Peticolas, "Polarized laser Raman studies of biological polymers," J. Chem. Phys., 51, 3993 (1969).

4. M. Tsuboi, T. Ikeda, and H. Shindo, "A velocity-gradient flow cell for Raman and emission spectroscopy of a deoxyribonucleic acid-drug solution," Chem. Pharm. Bull., 35, 4405 (1987).

5. T. W. Patapoff, G. A. Thomas, Y. Wang, and W. L. Peticolas, "Polarized Raman scattering from oriented single microcrystals of $d\left(A_{5} T_{5}\right)_{2}$ and d(pTpT)," Biopolymers, 27, 493 (1988).
6. T. Ueda, K. Ushizawa, and M. Tsuboi, "Depolarization of Raman scattering from some nucleotides of RNA and DNA," Biopolymers, 33, 1791 (1993).

7. J. M. Benevides, M. Tsuboi, A. H. J. Wang, and G. J. Thomas, Jr., "Local Raman tensors of double-helical DNA in the crystal: a basis for determining DNA residue orientations," J. Am. Chem. Soc., 115, 5351 (1993).

8. K. Fukushi and M. Kimura, "Raman spectra of halogen-substituted methanes in the liquid state: depolarization ratios of the totally symmetric vibration bands," J. Raman Spectrosc., 8, 125 (1979).

9. F. F. M. de Mul and J. Greve, "RAMPAC: a program for analysis of complicated Raman spectra," $J . R a$ man Spectrosc., 24, 245 ( 1993 ).

10. C. H. Wang and J. McHale, "Vibrational resonance coupling and the noncoincidence effect of the isotropic and anisotropic Raman spectral components in orientationally anisometric molecular liquids," $J$. Chem. Phys., 72, 4039 (1980).

11. V. M. Shelley and J. Yarwood, "The noncoincidence effect in N,N-dimethylformamide: a comparison of theoretical predictions and experimental results," Chem. Phys., 137, 277 (1989).

12. R. Letellier, M. Ghomi, and E. Taillandier, "Interpretation of DNA vibration modes, II: the adenosine and thymidine residues involved in oligonucleotides and polynucleotides," J. Biomol. Str. Dynamics, 4, 663 (1987).

13. G. J. Thomas, Jr., J. M. Benevides, S. A. Overman, T. Ueda, K. Ushizawa, M. Saitoh, and M. Tsuboi, "Polarized Raman spectra of oriented fibers of A DNA and B DNA: anisotropic and isotropic local Raman tensors of base and backbone vibrations," Biophys. J., 68, 1073 (1995).

14. S. N. Rao and P. Kollman, "On the role of uniform and mixed sugar puckers in DNA double-helical structures," J. Am. Chem. Soc., 107, 1611 (1985).

15. S. Brahms, V. Fritsch, J. G. Brahms, and E. Westhof, "Investigations on the dynamic structures of adenine- and thymine-containing DNA," J. Mol. Biol., 223, 455 (1992).

16. P. Kollman, J. W. Keepers, and P. Weiner, "Molecular-mechanics studies on d(CGCGAATTCGCG) ${ }_{2}$ and $\mathrm{dA}_{12} \cdot \mathrm{dT}_{12}$ : an illustration of the coupling between sugar puckering and DNA twisting," Biopolymers, 21, 2345 (1982).

17. R. Chandrasekaran and A. Radha, "Structure of poly (dA) - poly (dT)," J. Biomol. Str. Dynamics, 10, 153 (1992).

18. W. Saenger, Principles of Nucleic Acid Structure, Springer-Verlag, New York, 1984, pp. 58-63.

Received March 10, 1995

Revised May 29, 1995

Accepted June 8, 1995 